\title{
Las empresas de Norte de Santander y su perspectiva acerca de la seguridad y salud en el trabajo
}

\author{
Norte de Santander entreprises and \\ their perspectives about security and \\ health at work
}

Oscar Yesid Guerrero Jaimes Universidad de Pamplona, Norte de Santander.

Correo electronico: oscaryesid2004@gmail.com

Ruth Mayerly Guerrero Jaimes Autor de correspondencia Universidad de Pamplona, Norte de Santander.

Correo electronico:

Ruth.gerrero@unipamplona.edu.co 


\section{Resumen}

Colombia viene afrontando un proceso, de transición del programa de salud ocupacional, al sistema de gestión de seguridad y salud en el trabajo (SG-SST), acompañado con nuevas legislaciones en esta materia. El objetivo de esta investigación fue medir el grado de conocimiento, que tienen las empresas legalmente constituidas de Norte de Santander, con respecto a este tema de la seguridad laboral, con la finalidad de tener un panorama claro que pueda determinar las posibles estrategias que faciliten esta transición; Este nuevo SGSST obliga a los empleadores a realizar un reconocimiento permanente de las condiciones de trabajo que inciden en el bienestar, la seguridad y la salud de los trabajadores, permitiendo realizar las acciones de mejora. El no cumplimento de estas traerá sanciones económicas y hasta puede ocasionar el cierre definitivo de las empresas.

\section{Abstract}

Colombia has been facing a process of transition in the Health Occupational Program, in the system of security management and health at work (SG-SST), together with new legislation on this subject. The aim of this research is to measure the degree of knowledge that legally formed enterprises in Norte de Santander have got. In relation to this issue of work security, with the aim of having a clear path that may determine the possible strategies that facilitate this transition; this new SGSST make employers to do a permanent check on the working conditions that affect welfare, security and health on workers, thus allowing actions for improvement. Not complying with these tasks would bring economic sanctions even to the point of causing the final closing of the enterprises.

\section{Palabras \\ Claves:}

Salud ocupacional, gestión, seguridad, trabajo, legislación.

\section{Keywords:}

Occupational health, management, security, work, legislation. 


\section{Introducción}

La seguridad y salud en el trabajo constituyen una herramienta de gestión que permite el mejoramiento de la calidad de vida de los trabajadores en las organizaciones. Por tanto, es ampliamente utilizada en todos los sectores, generando grandes beneficios como prevención de enfermedades laborales, ambientes sanos de trabajo y disminución de costos generados por accidentes. Esta herramienta resulta muy efectiva cuando se centra en la generación de una cultura de seguridad engranada con productividad, desarrollo del talento humano, gestión de calidad, mejoramiento de procesos y condiciones adecuadas de puestos de trabajo.

Por otra parte, la salud ocupacional es un tema que se viene promoviendo hace varios años a nivel mundial, e instituciones como la Organización Internacional del Trabajo-OIT han desarrollado estrategias para que todos los países cumplan con los lineamientos que buscan mejorar la calidad de vida laboral de todos los trabajadores.

En Colombia este programa de salud ocupacional ha tomado un nuevo nombre: "Sistema de Gestión de Seguridad y Salud en el Trabajo". Entre otras cosas, la intención de este cambio va más allá de la simple denominación y busca más bien que el mismo deje de ser un documento que reposa en un archivo que nadie revisa, para convertirse en la guía de una política a la que se le hace seguimiento y mejora continua.

El Ministerio de Trabajo Nacional ha establecido los nuevos lineamientos para implementar el Sistema de Gestión de la Seguridad y Salud en el Trabajo, que debe ser implementado por todas las empresas del país. Por esta razón, es importante determinar el grado de información que poseen las empresas de Norte de Santander en cuanto a la seguridad y salud en el trabajo, así como acerca de la legislación y las sanciones previstas para las empresas que no lo implementen. Con otras palabras, es importante conocer qué significa la seguridad laboral para los empresarios. 


\section{Salud y seguridad en el trabajo}

Entre los países promotores del tema de la seguridad laboral, se destaca la Comunidad Europea, que en el año de 1989 dio los primeros pasos al respecto, implementando una importante normativa a nivel de seguridad laborar [1]. Concretamente, la Directiva de la Comunicad Europea del 12 de Junio de 1989 contiene los principios generales relativos a la prevención de riesgos laborales y su objetivo es "promover la mejora de la seguridad y salud de los trabajadores". La OIT es otro organismo que ha realizado esfuerzos para que los países implementen estas normas. En este sentido, las llamadas Estrategias Mundiales de Seguridad y Salud en el Trabajo propuestas por este organismo consisten principalmente en el desarrollo y mantenimiento de una cultura de prevención sobre seguridad y salud, y la introducción en Colombia de un enfoque sistemático de la gestión de la SST empieza por reconocer que: "Un Sistema de Gestión de la SST (SG-SST) puede ser concebido como una herramienta eficaz de prevención para hacer frente a los peligros y riesgos en el trabajo. Se basa en criterios, normas y resultados en materia de SST y, sobre todo, aspira a establecer mecanismos exhaustivos y estructurados para la acción, tanto para dirigentes como para trabajadores, cuando implementan medidas sobre seguridad y salud. Un Sistema de Gestión de la SST sigue un método lógico y progresivo para determinar qué es necesario hacer, la mejor manera de hacerlo, para supervisar los progresos, evaluar la calidad de las medidas adoptadas e identificar las áreas que deben ser mejoradas. Y, sobre todo, es un mecanismo que puede ser mejorado de manera constante y continua" [2].

El Sistema de Gestión de Seguridad y Salud en el Trabajo (SG-SST) se constituye así en una disciplina que trata de prevenir las lesiones y las enfermedades causadas por las condiciones de trabajo, además de velar por la protección y promoción de la salud de los empleados: "Tiene el objetivo de mejorar las condiciones laborales y el ambiente en el trabajo, además de la salud en el trabajo, que conlleva la promoción del mantenimiento del bienestar físico, mental y social de los empleados" [3]. 
A continuación se presentan algunos conceptos claves en seguridad y salud laboral:

Peligro: Se define como cualquier fuente, situación o acto que tenga el potencial de producir un daño en términos de una lesión o enfermedad, daño a la propiedad, al medio ambiente o una combinación de éstos. Más en concreto, este término se usa para describir algo presente en el lugar de trabajo que tiene el potencial de causar una lesión a los trabajadores, ya sea un accidente de trabajo o una enfermedad profesional.

Riesgo: Se entiende como una probabilidad de que ocurra un evento o de una exposición peligrosa y en consecuencia se presente en alguien una lesión o enfermedad severa.

Evaluación de riesgos: es el proceso para identificar los peligros derivados de las condiciones de trabajo. Se trata de un examen sistemático de todos los aspectos de las condiciones de trabajo para: identificar lo que pueda causar lesiones o daños, eliminar los peligros que puedan ser suprimidos, evaluar los riesgos que no se puedan eliminar inmediatamente y planificar la adopción de medidas correctoras.

Seguridad: Concepto de difícil definición derivado de la situación de estar "seguro", es decir, libre de cualquier daño o riesgo, aunque en la práctica es imposible conseguir esta situación completa. Por lo tanto, la seguridad se debe entender como un determinado nivel de riesgo que pueda considerarse como aceptable.

Salud: En relación con el trabajo no sólo incluye la ausencia de afecciones o enfermedades, sino también los elementos físicos y mentales directamente relacionados con el mismo, que pueden afectar negativamente a la salud. 
Enfermedad profesional: Es la dolencia contraída como resultado de una exposición durante un período de tiempo a factores (agentes químicos, físicos o biológicos) provenientes de la actividad laboral. Comprende cualquier dolencia crónica producida como resultado de un trabajo o actividad laboral. Este tipo de enfermedad se identifica al demostrar que es más frecuente en un determinado sector laboral que en la población general o que en otros grupos de trabajadores. Como ejemplo se encuentran las enfermedades respiratorias (por ejemplo, asbestosis o asma ocupacional), las enfermedades de la piel (por ejemplo, síndrome del túnel carpiano), los trastornos osteomusculares y el cáncer profesional.

Accidente laboral: Es un suceso puntual en el curso del trabajo que produce daño físico o mental. Puede ocurrir fuera de las instalaciones de la empresa o ser causado por terceros.

Prevención: Comprende todos los pasos o medidas adoptadas o previstas en todas las fases de la actividad de la empresa para evitar o reducir los riesgos laborales [1].

Los anteriores conceptos se aplican a cualquier tipo de trabajo, incluyendo sectores de la construcción, la agricultura, la industria manufacturera, la minería, la pesca, el sector servicios o la asistencia sanitaria; también incluyen a las empresas y trabajadores subcontratados. En cuanto a la seguridad y salud laboral, además del período de actividad de trabajo, se toma en cuenta el trayecto y el tiempo empleado en ir y venir del lugar de trabajo como parte de la jornada laboral. Los desplazamientos seguros constituyen, por tanto. una de las áreas que cubre la seguridad y salud laboral [1].

El nuevo sistema obliga a los contratantes a realizar un reconocimiento permanente de las condiciones de trabajo que inciden en el bienestar, la seguridad y la salud de los trabajadores, con el fin de realizar oportunamente las acciones de mejora. 
Este sistema nace de nuevos modelos laborales, en el marco de un mundo globalizado, que exige a las empresas brindar condiciones de seguridad óptimas, así como prevenir enfermedades y accidentes laborales.

Tabla1. Organismos especializados en SST a nivel mundial.

\section{Organización Internacional del Trabajo (OIT)}

Organizaciones de Europa (UE y EFTA). A este organismo pertenecen 11 miembros de la comunidad Europea.

Organizaciones de Europa (no UE ni EFTA) y Asia. A este organismo lo conforman 7 miembros de Asia y Europa.

Organizaciones de Norteamérica, Centroamérica y caribe. A este organismo lo conforman 29 miembros de América de Norte y Centroamérica.

Organizaciones de Sudamérica.

Constituido por 31 miembros.

\section{Organizaciones de África.}

Organismo conformado por 9 miembros.

Organizaciones de Oceanía y Extremo Oriente. Lo conforman 20 miembros.

Agencia Europea para la Seguridad y la Salud en el trabajo.

Agencia Europea para la Seguridad y la Salud e Higiene en el trabajo (ALASEHT).

Consejo Colombiano de Seguridad. 


\section{La seguridad en trabajo en Colombia}

El programa de salud ocupacional en Colombia ha afrontado cambios en los últimos años, incluido su nombre: pues, de "Sistema de seguridad laboral", cambio a "Sistema de Gestión de Seguridad y Salud en el Trabajo". Por otra parte, bajo este nombre se reconoce al documento maestro de la seguridad laboral en el trabajo documento que ha de ser una guía y carta de navegación para las empresas en cuanto a la seguridad laboral.

El Ministerio de Trabajo [4] plantea que el nuevo Sistema de Gestión de la Seguridad y Salud en el Trabajo debe ser implementado por todas las empresas del país, por quienes contratan personal en la modalidad de prestación de servicios (civil, comercial o administrativo), las organizaciones de economía solidaria y del sector cooperativo, así como por las empresas de servicios temporales.

La actual legislación en materia de riesgos laborales en Colombia obliga así a todos los empleadores y organizaciones a prevenir y controlar todos los riesgos laborales existentes en sus operaciones, de modo que Norte de Santander no es ajena a esta normativa.

Según el Ministerio del Trabajo [4], "la seguridad y la salud en el trabajo (SST) es una disciplina que trata de la prevención de las lesiones y enfermedades relacionadas con el trabajo, y de la protección y promoción de la salud de los trabajadores. Tiene por objeto mejorar las condiciones y el medio ambiente de trabajo. La salud en el trabajo conlleva la promoción y el mantenimiento del más alto grado de salud física y mental y de bienestar de los trabajadores en todas las ocupaciones".

En este contexto, la anticipación, el reconocimiento, la evaluación y el control de los peligros que surgen en el lugar de trabajo o dimanantes del mismo y que pueden poner en peligro la salud y el bienestar de los trabajadores son los principios fundamentales del proceso que rige la evaluación y gestión de los riesgos. También se deben tener en cuenta los posibles efectos en las comunidades vecinas y en el medio ambiente general:

“El proceso básico de aprendizaje sobre la reducción de los peligros y los riesgos 
es el origen de los principios más complejos por los que se rige la SST en la actualidad. Hoy por hoy, el hecho de que sea imperativo controlar una industrialización galopante y su necesidad de fuentes de energía sumamente e inherentemente peligrosas, como la utilización de la energía nuclear, los sistemas de transporte y unas tecnologías cada vez más complejas, ha conducido a la elaboración de unos métodos mucho más complejos de gestión y evaluación de los riesgos" [2].

Es claro, por otra parte, que en todos los ambientes de la actividad humana es preciso hallar un equilibrio entre los beneficios y los costos que suponen la asunción de riesgos. En el caso de la SST, este complejo equilibrio está influido por muchos factores, como el rápido progreso científico y tecnológico, la gran diversidad del mundo del trabajo y su continua evolución, y la economía. El hecho de que la aplicación de los principios de SST conlleve la movilización de todas las disciplinas sociales y científicas expresa claramente la complejidad de este ámbito.

El SG-SST, propuesto en el decreto 1443 de 2014 [5], el cual está basado en el ciclo PHVA (planificar, hacer, verificar y actuar), obedece, en efecto, a una serie de pasos lógicos y consecuentes, que comprenden desde el diseño de SG-SST (planear), pasando por el implementación y ejecución (Hacer), y terminando con una evaluación a través de auditorías (Verificar), con la finalidad de determinar las posibles desviaciones y fallas del mismo, para a continuación realizar un plan de acción que permita su eliminación o control (Actuar).

Partiendo de los expuesto anteriormente, el Ministerio del Trabajo emitió la resolución 1111 de 2017, con la que define los estándares mínimos del SGSST, para que las organizaciones que cumpla cabalmente, tengan lugares de trabajo seguros, libres de accidentes y enfermedades labores, en aras de mantener y preservar la integridad del trabajador y llegar a la excelencia laboral. Todo ello se reconocerá en una certificación que expedirá el mismo organismo a partir del año 2020. 


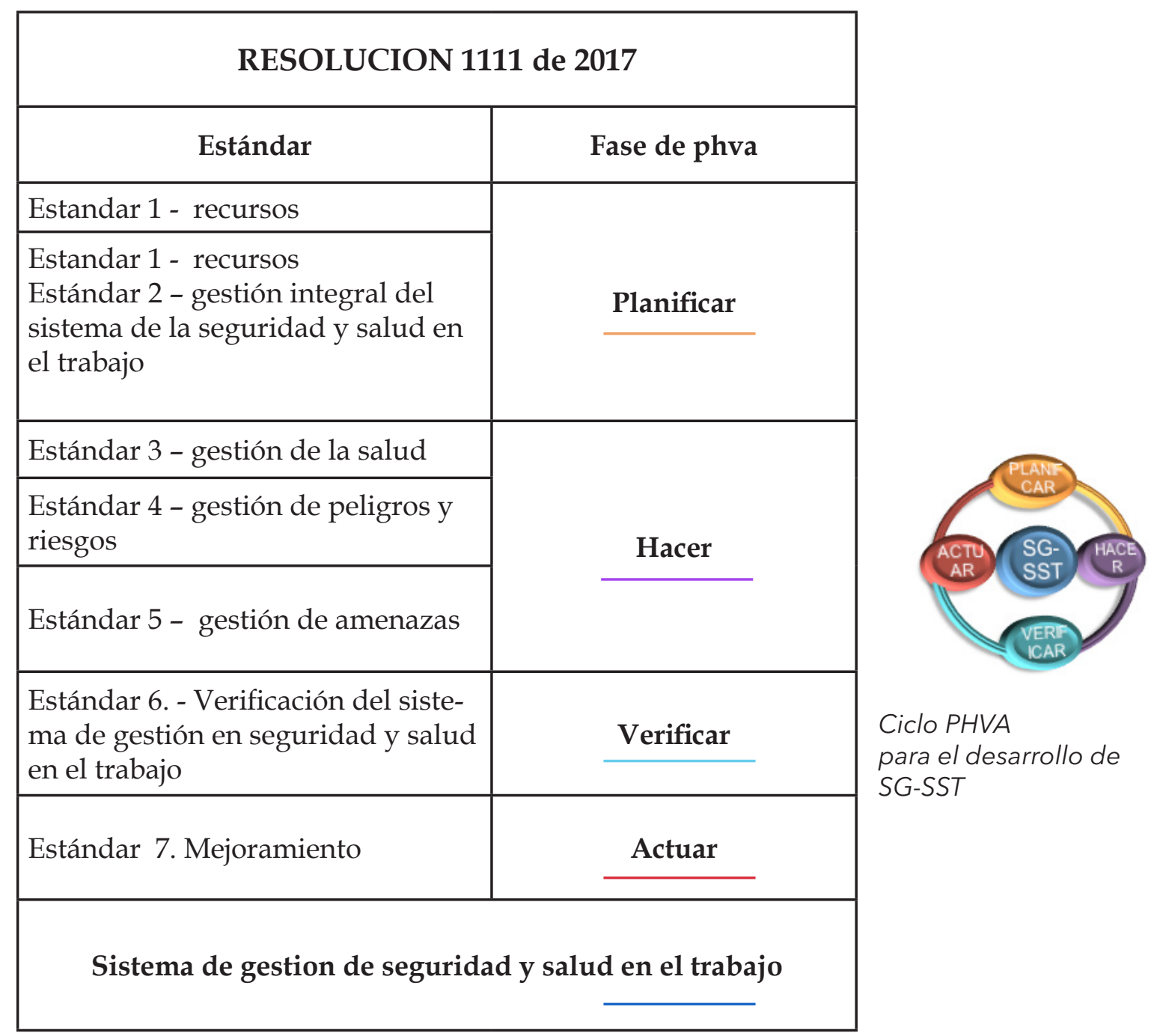




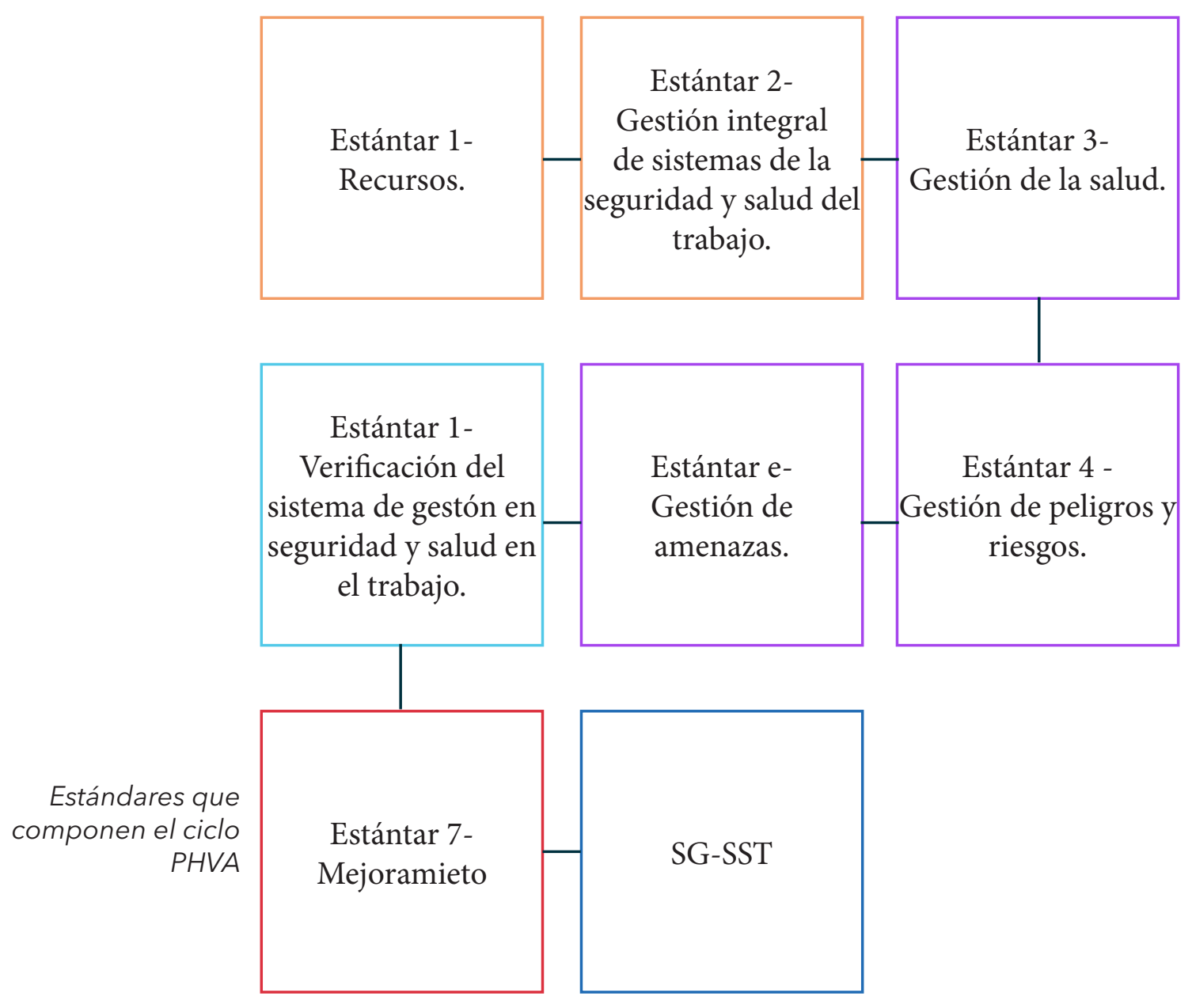




\section{Marco legal}

Como se ha reiterado, el Decreto 1443 de 2014 establece que la principal función del Sistema General de Riesgos Laborales es la promoción de la seguridad y salud en el trabajo y la prevención de los riesgos laborales, para evitar accidentes de trabajo y enfermedades laborales.

\section{Legislación internacional}

La Comunidad Andina, de la cual hace parte Colombia, adoptó en la Decisión 584 el "Instrumento Andino de Seguridad y Salud en el Trabajo", mediante el cual se establecen las normas fundamentales en materia de seguridad y salud en el trabajo que sirven de base para la gradual y progresiva armonización de las leyes y los reglamentos que regulen las actividades laborales que se desarrollan en cada uno de los países miembros. Esta decisión deben servir también para impulsar en tales países la adopción de directrices sobre sistemas de gestión de la seguridad y la salud en el trabajo, así como para propiciar el establecimiento de un sistema nacional de seguridad y salud en el trabajo. En este marco, el artículo 56 del Decreto 1295 de 1994, sobre la prevención de los riesgos laborales, establece que una de las responsabilidades del Gobierno Nacional de Colombia, consiste en expedir las normas técnicas reglamentarias tendientes a garantizar la seguridad' de los trabajadores y de la población en general, en la prevención de accidentes de trabajo y enfermedades laborales.

Además, la Organización Internacional del Trabajo-OIT publicó en el año 2001, las 
directrices relativas a los sistemas de gestión de la 'seguridad y la salud en el trabajo (ILO-OSH, 2001), las cuales, debido a su enfoque tripartito, se han convertido en un modelo ampliamente utilizado para elaborar normas nacionales.

El siguiente cuadro resume, finalmente, la historia de la legislación de la seguridad en el trabajo.

\begin{tabular}{|c|c|c|}
\hline Normas & Año & Descripción \\
\hline Ley 57 & 1915 & $\begin{array}{l}\text { Hace referencia a la reglamentación de los accidentes de } \\
\text { trabajo y las enfermedades profesionales. Históricamente } \\
\text { establece la primera y estructurada definición de Accidente de } \\
\text { Trabajo. }\end{array}$ \\
\hline Ley 90 & 1946 & $\begin{array}{l}\text { Por la cual se establece el seguro social obligatorio y se crea el } \\
\text { Instituto Colombiano de Seguros Sociales. Entidad Promotora } \\
\text { de Salud (EPS), Instituto Prestador de Salud (IPS), Cesantías, } \\
\text { Pensiones y la Aseguradora de Riesgos Profesionales. }\end{array}$ \\
\hline $\begin{array}{l}\text { Decreto } \\
\text { Ley } 2663\end{array}$ & 1950 & $\begin{array}{l}\text { Se expide el Código Sustantivo del Trabajo, estableciendo } \\
\text { normas relativas a la Salud Ocupacional. }\end{array}$ \\
\hline Ley 9 & 1979 & $\begin{array}{l}\text { Nace el término de Salud Ocupacional. } \\
\text { Se dictan las normas generales para preservar, conservar y } \\
\text { mejorar la salud de los individuos en sus ocupaciones. }\end{array}$ \\
\hline $\begin{array}{l}\text { Resolución } \\
2400\end{array}$ & 1979 & $\begin{array}{l}\text { Se establecen disposiciones sobre vivienda, higiene y } \\
\text { seguridad en los establecimientos de trabajo }\end{array}$ \\
\hline $\begin{array}{l}\text { Decreto } \\
586\end{array}$ & 1983 & Se crea el comité Nacional de Salud Ocupacional \\
\hline $\begin{array}{l}\text { Decreto } \\
614\end{array}$ & 1984 & $\begin{array}{l}\text { Por el cual se determinan las bases para la organización y } \\
\text { administración de Salud Ocupacional en el país }\end{array}$ \\
\hline $\begin{array}{l}\text { Resolución } \\
2013\end{array}$ & 1986 & $\begin{array}{l}\text { Se reglamenta la organización y funcionamiento de los } \\
\text { comités de Medicina, Higiene y Seguridad Industrial en los } \\
\text { lugares de trabajo. }\end{array}$ \\
\hline $\begin{array}{l}\text { Resolución } \\
\quad 1016\end{array}$ & 1989 & $\begin{array}{l}\text { Se reglamenta la organización, funcionamiento y forma de los } \\
\text { programas de Salud Ocupacional que deben desarrollar los } \\
\text { patronos o empleadores en el país }\end{array}$ \\
\hline $\begin{array}{l}\text { Resolución } \\
\quad 1075\end{array}$ & 1992 & $\begin{array}{l}\text { Se reglamentan actividades en materia de Salud Ocupacional } \\
\text { Se considera que es competencia del Ministerio de Trabajo } \\
\text { y Seguridad Social, establecer medidas tendientes a que el } \\
\text { trabajo se realice dentro de condiciones apropiadas. }\end{array}$ \\
\hline
\end{tabular}




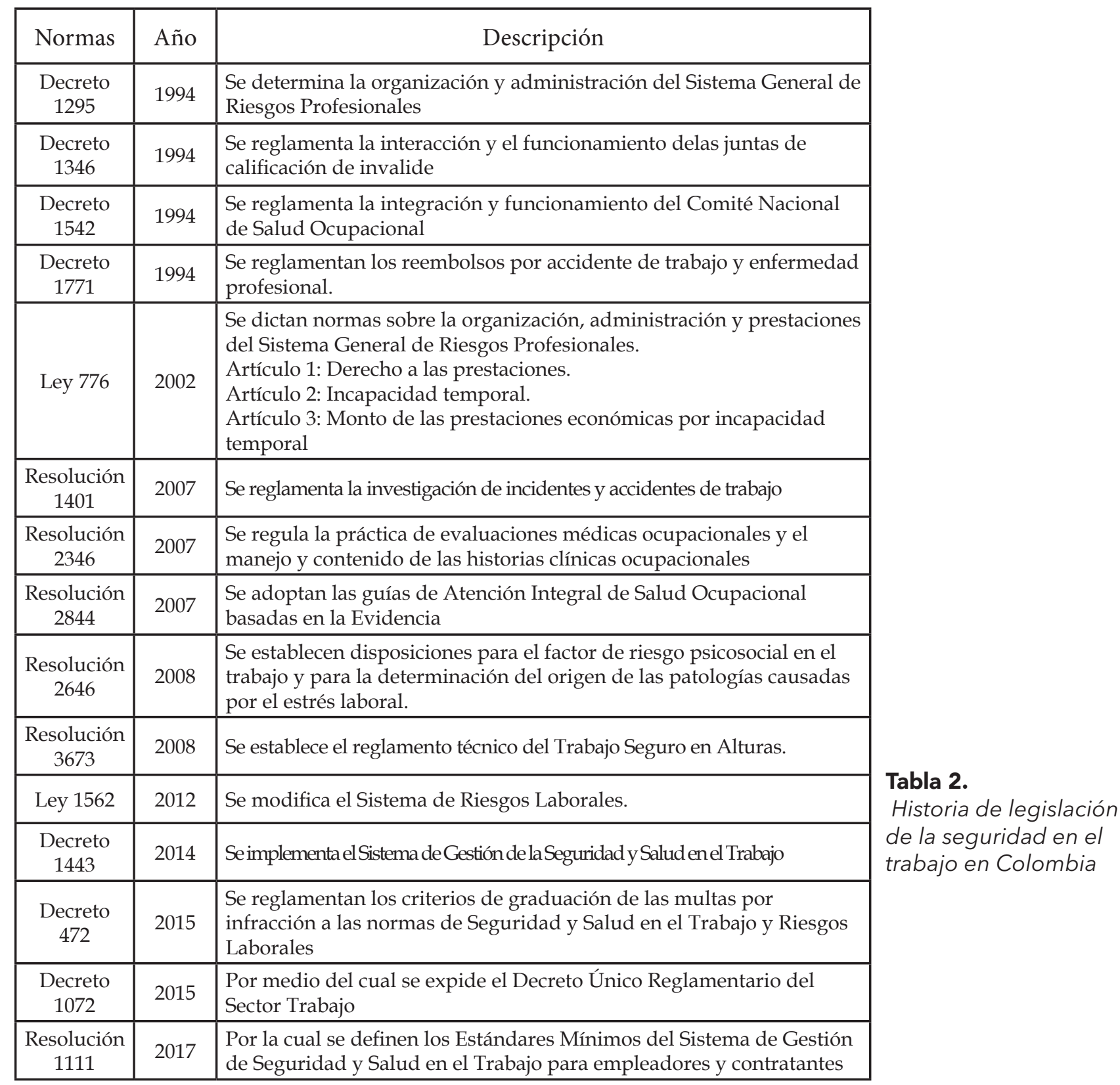




\section{METODOLOGÍA}

En este artículo se realizó una revisión bibliográfica acerca de la seguridad y salud en el trabajo, lo cual permitió adoptar un instrumento para recopilar información y plantear los resultados. [5]

Para lo anterior se ha tenido en cuenta lo indicado en la Organización Internacional del Trabajo - OIT, la cual público en el año 2001, las Directrices relativas a los sistemas de gestión de la 'seguridad y la salud en el trabajo (ILO-OSH 2001) las cuales, debido a su enfoque tripartito, se han convertido en un modelo ampliamente utilizado para elaborar normas nacionales. [2] 


\section{Resultados y discución}

Después de aplicar el instrumento que permitió recopilar parte de la información planteada para el cumplimiento de los objetivos, se determinó que:

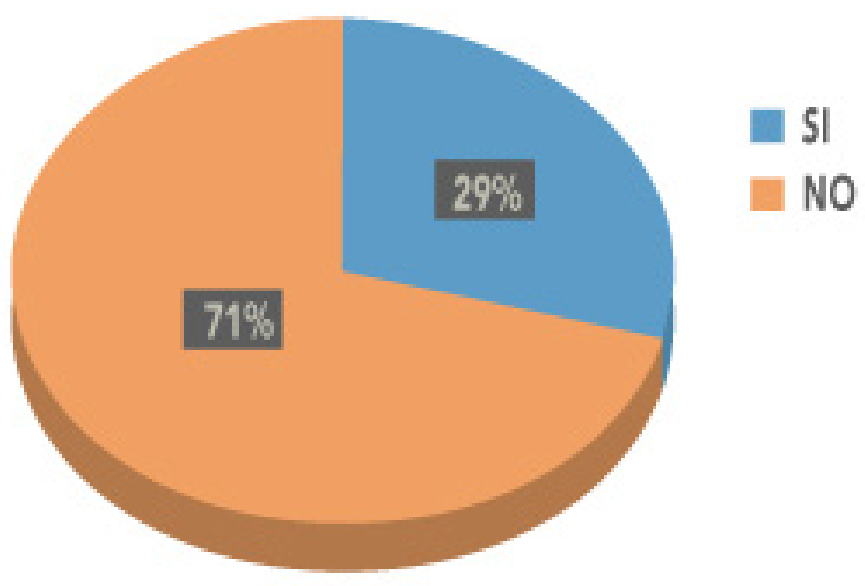

Figura 1.

- E1 71\% de las empresas legalmente constituidas del departamento de Norte de Santander desconocen la existencia de legislación en seguridad y salud en el trabajo. De modo que solo el $29 \%$ de las empresas legalmente constituidas de este departamento tiene conocimiento de esta nueva legislación. Esto significa que un alto porcentaje de las empresas en el Norte de Santander tiene poca idea del tema de seguridad laboral.

- Al indagar a las mismas empresas respecto a si cuentan con un programa de salud ocupacional o un sistema de gestión, se encontró que el $79 \%$ no lo posee, en tanto que el restante $21 \%$ sí cuenta con el programa de salud ocupacional o sistema de gestión de seguridad y salud en el trabajo. 


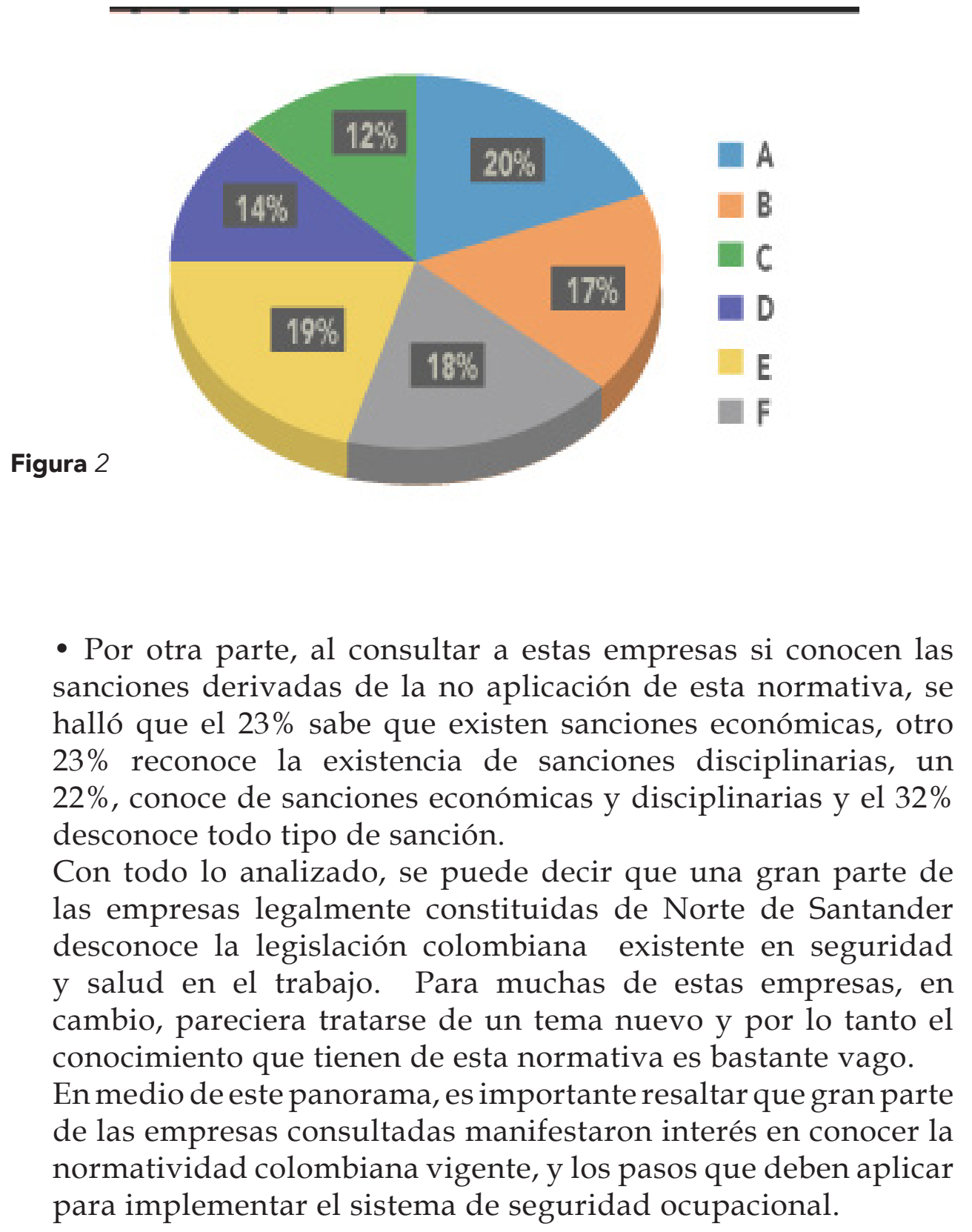




\section{Conclusión}

Desafortunadamente, a pesar de que nuestro país dispone de una legislación avanzada en materia de seguridad laboral, se siguen presentando críticas a la aplicación del sistema de gestión de seguridad y salud en el trabajo por su ineficiencia y falta de mayor trabajo en prevención. Entre las razones que posibilitan esta situación están: el desconocimiento de la ley, especialmente a nivel de los trabajadores; los costos del sistema de gestión de seguridad y salud en el trabajo; y la falta de conciencia para la prevención de los riesgos profesionales en las empresas.

Por lo anterior, es claro que la mayoría de los trabajadores formales de Norte de Santander se encuentran expuestos a los riesgos propios de sus labores sin ningún control, poniendo en peligro su integridad física, mental y salud laboral. A esto se suma que la cultura de la prevención de los riesgos y peligros en los puestos de trabajo en las empresas legalmente constituidas de Norte de Santander, es muy baja, debido al desconocimiento de la normativa, falta de asesoría y campañas de prevención. Incluso, existen empresas que aún no tienen el programa de salud ocupacional y desconocen la legislación actual; por lo tanto desconocen también las sanciones en las que pueden incurrir por el incumplimiento de las mismas. Hay mucho por hacer en este tema de salud y seguridad laboral, pero todo ello implica un trabajo que se debe realizar en conjunto, incluyendo a todas las entidades involucradas: Estado, empresas y aseguradoras de riesgos laborales (ARL). Se sugiere comenzar por la realización de campañas de información con respecto a este tema, que es muy importante para la salud y bienestar de los trabajadores y para la tranquilidad de las empresas. 


\section{Referencias Bibliográficas}

1. Nunes "Aspectos generales de seguridad y salud en el trabajo $($ SST)" (2013), disponibles en: oshwiki.eu/wiki/Aspectos_generales_ de_seguridad_y_salud_en_el_trabajo_(SST)

2. Organización Internacional del Trabajo.www.ilo.org. 2011 Disponible en: http://www.ilo.org/wcmsp5/groups/public/@ed_ protect/@protrav/@safework/documents/publication/wcms_154127. pdf

3. Peña, "En qué consiste el Sistema de Gestión de la Seguridad y Salud en el Trabajo (SG-SST)?" Director Sistemas de Gestión Codelca (2016) Disponible en: /www.isotools.org/2016/09/06/consiste-sistemagestion-la-seguridad-salud-trabajo-sg-sst/

4. Ministerio del Trabajo (2016). Disponible en: http://www. mintrabajo.gov.co/febrero-2016/5796-mipymes-contaran-con-guiapara-seguridad-y-salud-en-el-trabajo.html

5. Decreto 1443 de 2014, Disponible en: http:/ / www.alcaldiabogota. gov.co/sisjur/normas/Norma1.jsp?i=58841 


\section{Este artículo se cita}

O. Y. Guerrero.,y R. M. Guerrero, "Las empresas de Norte de Santander y su perspectiva acerca de la seguridad y salud en el trabajo", Investigación e Innovación en Ingenierias, vol. 5, $\mathrm{n}^{\circ} .2$, pp. 26-45, 2017 